\title{
Diffuse melanoma of the choroid
}

\author{
DEVRON H. CHAR, R. DUDLEY STONE, J. BROOKS CRAWFORD, \\ ROBERT WALLYN, ALEXANDER R. IRVINE, GEORGE F. HILTON, \\ HOWARD SCHATZ, ARIAH SCHWARTZ, AND LAWRENCE LONN \\ From the Ocular Oncology Unit, the Department of Ophthalmology, and the Francis $I$. \\ Proctor Foundation, University of California, San Francisco, USA
}

SUMMARY An 81-year-old woman with good vision was followed up with the diagnosis of a minimally active melanoma. This tumour was eventually found to be a diffuse melanoma with extraocular extension. Diffuse melanomas of the uvea are difficult to diagnose, have frequent and early extrascleral extension, and have a poorer prognosis than most melanomas of the uveal tract.

Diffuse melanomas of the choroid involve at least $25 \%$ of the uveal tract and have minimal elevation (Parsons, 1904). These tumours are particularly treacherous, since they are difficult to diagnose, undergo early and frequent extrascleral extension, and have a higher incidence of metastatic disease than most other forms of choroidal melanoma (Reese and Howard, 1967; Font et al., 1968).

In some ocular oncology centres serial examinations without intervention are performed on patients with slightly raised pigmented choroidal mass lesions until growth is detected (Char and Hogan, 1977; Shields, 1977; Char, 1978). We present a report on a patient suspected of having either a large choroidal naevus or a choroidal melanoma. When growth was detected, the lesion was eventually shown to be diffuse choroidal melanoma with extrascleral extension. This patient presents some of the problems associated with making the correct diagnosis in pigmented choroidal masses, points out the risks of following up such patients, and emphasises the necessity to consider diffuse melanoma in those patients with slightly raised pigmented lesions of large diameter of the uveal tract.

\section{Case report}

The patient was an 82-year-old Caucasian female who was initially examined by her local ophthalmologist because of sharp pains of recent onset in the right eye. The patient's systemic and ocular past medical history was essentially normal.

On initial evaluation in the Ocular Oncology

Correspondence to Devron H. Char, MD, S-315, University of California, San Francisco, California 94143, USA.
Unit in November 1977 her visual acuity in the right eye was 20/25 (6/7.5). Examination of the anterior segment showed nothing abnormal. Examination of the posterior pole of the eye revealed a pigmented, slightly raised $(<2.5 \mathrm{~mm})$ choroidal lesion extending from approximately 5.30 to 8.30 o'clock and involving both the posterior and anterior fundus. There were multiple drusen over the surface of this rounded lesion, without orange pigmentation or subretinal fluid. No scotoma could be demonstrated with either a 1-4-E or a IV-4-E isoptre on Goldmann perimetry.

A fluorescein angiogram was nondiagnostic. The affected area of the choroid showed minimal hyperfluorescence without tumour vessels, hot spots, or subretinal leakage. B-scan ultrasonography showed a tumour mass of approximately $2 \mathrm{~mm}$ in elevation with equivocal choroidal excavation.

This lesion was thought to be minimally active choroidal melanoma, and after discussion with the patient it was decided to follow her up with serial examinations.

The lesion appeared to remain stable until about 11 months after the patient's initial visit, when increased elevation was observed on clinical examination. The overall diameter of the lesion was unchanged, but in its central portion a relatively nonpigmented mass was noted to be approximately $5 \mathrm{~mm}$ in elevation. Examination of this lesion by ultrasonography and computerised tomography suggested extraocular extension (Figs. 1 and 2). A radioactive phosphorus uptake test was strongly positive, and during the performance of this test a large extrascleral extension was observed which arose from the posterior, nonelevated portion of 


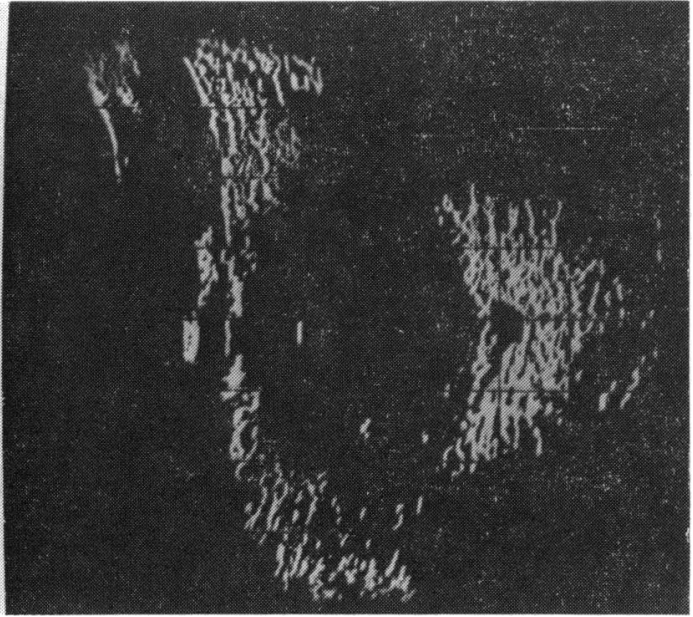

Fig. 1 CT scan showing extraocular extension

the tumour mass. An extensive search for metastases was negative, and an orbital exenteration was performed 2 days later.

Microscopic examination showed a flat diffuse tumour extending from the ciliary body to the posterior pole, equal to approximately $1 / 3$ the circumference of the globe (Fig. 3). The tumour was composed primarily of large, bizarre epithelioid cells; there was no break through Bruch's membrane. Near the posterior margin of the tumour distant from the area of elevation observed clinically there was an extrascleral extension.

The patient has done well, without evidence of metastatic disease, in the 12 months since surgery.

\section{Discussion}

Fuchs (1882) described diffuse malignant melanomas of the uveal tract. Since then approximately 130 patients with diffuse melanomas have been reported (Fuchs, 1882; Ewetzky, 1898; Kipp, 1903-05; Parsons, 1904; Cargill and Mayou, 1907; Alexander and Shea, 1964; Reese and Howard, 1967; Spaulding et al., 1967; Font et al., 1968; Machemer, 1968). Font et al. (1968) have enumerated criteria for the diagnosis of diffuse melanoma: involvement of at least $\frac{1}{4}$ of the uveal tract; tumour thickness between 3 and $7 \mathrm{~mm}$ in height; and involvement of the posterior uvea if the main tumour is in the iris or pars plicata.

Diffuse melanomas of the uveal tract are difficult to diagnose. Font et al. (1968) reported that $40 \%$ of their cases were not considered to have tumours before enucleation. Often the presentation of these tumours may simulate absolute glaucoma, uveitis, or recurrent scleritis. In the present case none of

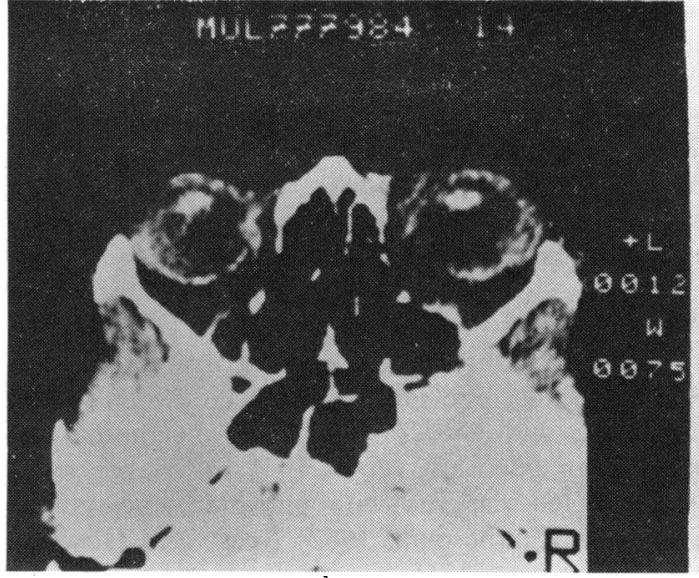

Fig. 2 B-scan ultrasound showing extraocular extension of a choroidal melanoma

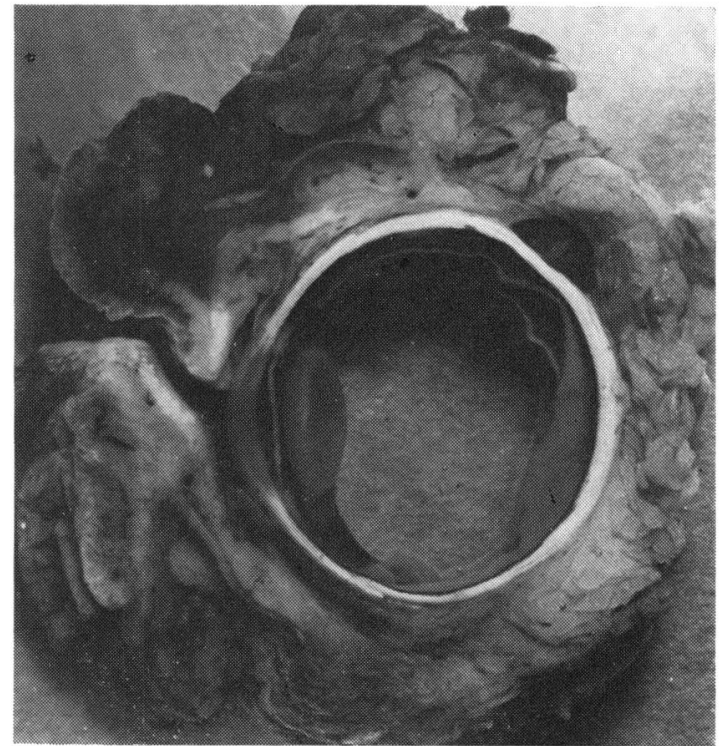

Fig. 3 Exenteration specimen showing extraocular extension of the diffuse melanoma

these findings was observed, and the patient's visual acuity was excellent. Reese and Howard (1967) described the typical diffuse choroidal melanoma as having surface drusen and finger-like projections; neither of these signs clearly differentiates a choroidal naevus from this malignant tumour. The vast majority of choroidal naevi are less than $6 \mathrm{~mm}$ in diameter and $1.5 \mathrm{~mm}$ in height; in lesions larger than this the possibility of a diffuse melanoma should be raised (Gass, 1977). While we have abandoned the routine use of the radioactive phosphorus uptake test in the evaluation of choroi- 
dal melanoma patients, perhaps in this group of subjects, those with minimally raised large pigmented choroidal lesions, it may be useful (Reese and Howard, 1967). In the present case, and 2 patients reported by Reese and Howard (1967), the radioactive phosphorus uptake results were strongly positive. However, in other cases of diffuse and localised choroidal melanomas false negative and false positive results have been observed (Alexander and Shea, 1964; Zakov et al., 1978).

It is important to make the correct diagnosis early in diffuse melanoma. Between 39 and $53 \%$ of patients with this form of uveal melanoma had extraocular extension versus $13 \%$ in a large series of unselected patients with choroidal melanomas (Starr and Zimmerman, 1962; Reese and Howard, 1967; Font et al., 1968). These patients tend to have a generally more malignant cell type on histological examination and a lower survival rate than most other types of choroidal melanoma (Font et al., 1968).

This work was supported in part by a grant from That Man May See and by NIH grants EYO2072, EYO1441, and EYO1759. Dr Char is a recipient of an NIH Research Career Development Award KO4EYO0117.

\section{References}

Alexander, R. L., and Shea, M. (1964). Malignant melanoma en plaque. Archives of Ophthalmology, 72, 498-500.

Cargill, L. V., and Mayou, S. (1907). A case of flat sarcoma of the choroid. Transactions of the Ophthalmological Societies of the United Kingdom, 27, 149-155.

Char, D. H. (1978). The management of small choridal melanomas. Survey of Ophthalmology, 22, 377-386.
Char, D. H., and Hogan, M. J. (1977). The management of small elevated pigmented choroidal lesions. British Journal of Ophthalmology, 61, 54-58.

Ewetzky, T. (1898). Weitre Studien über intraoculäre Sarkome. Albrecht von Graefes Archiv für Ophthalmologie, 45, 563-589.

Font, R. L., Spaulding, A. G., and Zimmerman, L. E. (1968). Diffuse malignant melanoma of the uveal tract: A clinicopathologic report of 54 cases. Transactions of the American Academy of Ophthalmology and Otolaryngology, 72, 877-895.

Fuchs, E. (1882). Das Sarcom des Uvealtractus, p. 116. Braumuller: Vienna.

Gass, J. D. M. (1977). Problems in the differential diagnosis of choroidal nevi and malignant melanomas. Transactions of the American Academy of Ophthalmology and Otolaryngology, 83, 19-48.

Kipp, C. J. (1903-05). Two cases of melanosarcoma of the chorid. Transactions of the American Ophthalmological Society, 10, 432-438.

Machemer, R. (1968). Zur Pathogenese Des Flächenhaften Malignen Melanoms. Klinische Monatsblätter für Augenheilkunde, 148, 641-652.

Parsons, J. H. (1904). Diffuse sarcomata of the uveal tract. Archives of Ophthalmology, 33, 101-112.

Reese, A. B., and Howard, G. M. (1967). Flat uveal melanomas. American Journal of Ophthalmology, 64, 10211028.

Shields, J. A. (1977). Current approaches to the diagnosis and management of choroidal melanomas. Survey of Ophthalmology, 21, 443-463.

Spaulding, A. G., Green, W. R., and Font, R. L. (1967). Ring-shaped malignant limbal tumor. Archives of Ophthalmology, 77, 76-80.

Starr, H. J., and Zimmerman, L. E. (1962). Extrascleral extension and orbital recurrence of malignant malanomas of the choroid and ciliary body. International Ophthalmology Clinics, 2, 369.

Zakov, Z. N., Smith, T. R., and Albert, D. M. (1978). False-positive 32P uptake test. Archives of Ophthalmology, 96, 2240-2243. 